\title{
Implementation with State Dependent Feasible Sets and Preferences: A Renegotiation Approach*
}

Luis C. Corchón

\author{
Matteo Triossi \\ Department of Economics. Universidad Carlos III. \\ c/ Madrid 126, Getafe. 28903 Madrid, Spain.
}

June 13, 2005

\begin{abstract}
In this paper we present a model of implementation when the feasible set and preferences are state dependent based on the idea that agents renegotiate unfeasible allocations. We characterize the maximal set of Social Choice Correspondences that can be implemented in a class of renegotiation functions that do not reward agents for unfeasibilities.
\end{abstract}

${ }^{*}$ We thank Carmen Beviá for very useful comments. The paper was presented in the III Workshop of Social Choice held in Malaga, June 2005. We are indebted to the audience for very useful comments, specially to Asher Wolinski. We acknowledge financial support from CAICYT under project PB BEC2002-02194 and from Fundación BBVA. 


\section{Introduction}

Since the classical papers of Hurwicz in the early seventies, a great deal of attention has been devoted to the problem of implementing social choice rules when preferences are state dependent (see, e.g. Jackson [2000] for a survey). In contrast, very few contributions have dealt with the problem of implementing social choice rules when the set of feasible outcomes is state dependent: Hurwicz et al., 1995, Tian (1993), Tian and Li (1995), Hong (1995), (1996), (1998), Serrano and Vohra (1997) and Dagan et al. (1999). In this case it is impossible to guarantee the feasibility of mechanisms (see, e.g. Hurwicz et al. (1995)). Thus, we have to describe how society -and/or the planner- deals with unfeasible allocations. All these papers design a state dependent mechanism in which the planner can ex-post verify if players are exaggerating endowments or technological capabilities (i.e. by asking to put endowments on the table). If infeasibility occurs, players expect a serious punishment.

We have several reservations about this approach: The assumptions of ex-post verification of exaggeration only and a serious punishment if infeasibility arise are rather extreme. Moreover, it is not clear how to proceed without them. This approach also produces a curious asymmetry between mechanisms coping with state dependent preferences ("demand") and mechanisms coping with state dependent endowments ("supply"). The former are state independent but the latter are state dependent. Finally, the implementing mechanisms are even hard to describe so it may be costly to used them.

In this paper we present a general model of implementation with renegotiation when the set of feasible outcomes and preferences are state dependent. We model the social process which transforms unfeasible allocations into feasible ones by means of a reversion function. This concept originates in Maskin and Moore (1999) and has been developed by Jackson and Palfrey (2001). In these papers the reversion function for-

malized the process of renegotiation by means of which agents trade the goods allocated 
by the mechanism or veto some feasible allocations. In our case the reversion function represents the way in which society deals with unfeasible allocations: when facing infeasibility, agents just renegotiate case by case taking advantage of the information that is available on the spot. ${ }^{1}$ Consequently, the properties that we impose on the reversion function are very different from those assumed by the earlier literature.

We assume that agents know the reversion function and that they are interested only in reverted allocations. So the reversion function induces new preferences on the whole outcome set (this the "translation principle" in Maskin and Moore [1999]). Such preferences are state dependent even if primitive preferences are not. Thus, implementation when the feasible set is state dependent reduces to the case of implementation when only preferences are state dependent. However as remarked by Maskin and Moore, "results from the standard literature are too abstract to give a clear indication of how serious a constraint renegotiation is...". In our paper we study Nash implementation by simplicity but our approach can be applied to other solution concepts.

We focus our attention in a class of reversion function in which should an infeasibility arise at least one agent is made worse off. We call this a non-rewarding reversion function. Reversion functions considered before do not fall into this class because they assume that agents are made better off by renegotiating. The difference is explained by the fact that in their case, renegotiation comes from the inability of the mechanism to stop agents from reaching mutually beneficial trades. In our case renegotiation arises from the physical impossibility to carry out the intended plans so somebody has to make a sacrifice to achieve feasibility. An extreme case of a non-rewarding reversion

\footnotetext{
${ }^{1}$ Renegotiation may be channelled by institutions or may be totally free. A striking example is that of a legal system. Once infractions are detected there are institutions designed to punish transgressors and to restore feasibility. In our case we can think of the feasible set including not only the properly feasible allocations but also all punishments and additional devices that can be administrated by the designed institutions as well as the delays that may occur.
} 
function is when should an infeasibility arise, all agents are punished such that they prefer any allocation without punishment to the situation in which they are punished. This strong form of punishment -that we will call generalized severe resembles the one assumed in the previous literature but in our case it only serves an instrumental role: we show that in the class of non-rewarding reversion functions, the generalized severe reversion function implements the largest class of social choice rules (Proposition 1).

An easy adaptation of the classical result shows that monotonicity when reverted preferences are given by the generalized severe reversion function is a necessary and almost sufficient condition of implementation in Nash equilibrium (Remark 1). Our first task is to characterize this property. It is shown to be equivalent to a weak form of unanimity and a generalized form of contraction consistency (Proposition 2). The former property is satisfied by most social choice rules. The latter assumption is similar to Nash's independence of irrelevant alternatives but in our case the assumption refers to the feasible set and not necessarily to the utility possibility set.

Next, we apply the previous result to several frameworks and compare our findings with the earlier literature. In the case of exchange economies weak unanimity is trivially satisfied by any individually rational social choice rule. We show that the Constrained Walrasian rule satisfies generalized contraction consistency and thus it is implementable in our set up (Proposition 3). However the individual rationality requirement which in Hurwicz et al. (1995) is necessary and sufficient for feasible implementation is not sufficient for implementation in our framework. The reason is that the previous literature not only assumed that players never exaggerate their endowments but they never use messages designed for different state of the world even such messages would lead to an outcome which is feasible in the actual state of the world. We turn our attention to bargaining problems. We show that if the disagreement point is not state dependent the Nash Bargaining solution is implementable with a non-rewarding renegotiation function (Proposition 4). This agrees with the findings of Serrano (1997) and Naeve (1999). 
We also show that the Kalai-Smorodinski solution is not implementable. Finally we consider the taxation problem in which the mechanism has to collect a given amount of taxes. We find a negative result, namely that a taxation method is implementable if and only if it is a serial dictatorship, i.e. agents can be arranged such that the any agent pays the minimal tax compatible with the following agents to be able to complete the required amount. This negative result contrast with the permissive results obtained by Dagan et al. (1999). The difference between our approaches is that in their case the report sent by agents matters for the renegotiation and in our case it does not. Our result serves to highlight the negative consequences of disregarding reports (i.e. a fiscal amnesty) even if all agents show up the whole endowment.

\section{The model}

In this section we provide the main definitions. Let us first describe the environment.

Let $I=\{1, \ldots, n\}$ be the set of agents. Let $\omega_{i}$ be type of $i$ and $\Omega_{i}$ be agent $i$ 's type set. Let $\Omega \subset \prod_{i=1}^{n} \Omega_{i}$ be the set of all possible states of the world. Each $\omega \in \Omega$ is characterized by a list of individual outcome sets $\left(X_{1}(\omega), \ldots, X_{n}(\omega)\right)$, a feasible set $A(\omega) \subset$ $\prod_{i=1}^{n} X_{i}(\omega) \equiv X(\omega)$ and a preference profile $R(\omega)=\left(R_{1}(\omega), \ldots, R_{n}(\omega)\right)$. The outcome set of $i$ might include sanctions that can be charged to $i$ and other constraints like individual rationality, etc. $A(\omega)$ contains all feasible allocations including punishments that arise in state $\omega$. Set $A \equiv \bigcup_{\omega \in \Omega} A(\omega)$. Let $a=\left(a_{1}, \ldots, a_{n}\right) \in A$ be an allocation also written $\left(a_{i}, a_{-i}\right)$. Let $A_{i}(\omega)=\left\{a_{i} \in X_{i}(\omega): \exists a_{-i}\right.$ such that $\left.\left(a_{i}, a_{-i}\right) \in A(\omega)\right\}$ be agent $i$ 's feasibility constraint. Observe that $A(\omega)=\bigcap_{i=1}^{n}\left\{a: a_{i} \in A_{i}(\omega)\right\} . R_{i}(\omega)$ is a preference relation on $X_{i}(\omega)$, a complete, reflexive and transitive binary relation on $X(\omega) . P_{i}(\omega)$ denotes the corresponding strict preference relation. Let $L_{i}(a, \omega)=\left\{x \in A(\omega): a R_{i}(\omega) x\right\}$ be agent $i$ 's lower contour set of $a_{i}$. Let $\Re_{i} \equiv \cup_{\omega_{i} \in \Omega_{i}} R_{i}\left(\omega_{i}\right)$ be the set of $i$ 's admissible preferences relations. Set $\Re=\prod_{i=1}^{n} \Re_{i}$. With abuse of notation, for every profile 
of preferences $R \in \Re$ we will denote by $R$ also the preference profile that $R$ induces on $A$.

A correspondence $F: \Omega \rightarrow A$ such that $F(\omega) \subset A(\omega)$ for all $\omega \in \Omega$ will be called a Social Choice Rule (SCR for brevity).

A mechanism is a pair $(M, g)$ where $M \equiv \prod_{1}^{n} M_{i}$ is the message space and $g: M \rightarrow A$ is the outcome function. $M_{i}$ denotes agent $i$ 's message space. Let $m=\left(m_{1}, . ., m_{n}\right) \in M$, be a list of messages also written $\left(m_{i}, m_{-i}\right)$. Given $\omega \in \Omega$, a mechanism $(M, g)$ induces a game $(M, g, R(\omega))$.

A message profile $m^{*} \in M$ is a Nash equilibrium for $(M, g, R(\omega))$ if, for all $i \in I$ $g\left(m^{*}\right) R_{i}(\omega) g\left(m_{-i}^{*}, m_{i}\right)$ for all $m_{i} \in M_{i}$.

$N E(M, g, R(\omega))$ will denote the set of allocations that are yielded by all Nash equilibria for $(M, g, R(\omega))$.

The mechanism $(M, g)$ implements $F$ in Nash equilibrium if, for all $\omega \in \Omega N E(M, g, R(\omega))=$ $F(\omega)$.

\section{Reversion functions}

Since outcomes that are feasible in some state may be unfeasible in others, we have to describe how society deals with unfeasible allocations. We assume that if an allocation is unfeasible it is transformed into a feasible one by a process that might involve delays (because renegotiation takes time), penalties to some individuals, etc. This systematic way in which the reallocation process takes place will be called a reversion function. ${ }^{2}$

\footnotetext{
${ }^{2}$ The case in which there may be several renegotiation functions is studied by Amorós (2004)
} 
This reallocation may correspond to a "free-market renegotiation" or to a process where the planner applies some kind of punishment or a bankruptcy rule. Formally:

Definition 1. A reversion function is a map $h: A \times \Omega \rightarrow A$ such that for each $\omega \in \Omega$, i) $h(a, \omega) \in A(\omega) \forall a \in A$ and ii) If $a \in A(\omega), h(a, \omega)=a$.

A reversion function always yields feasible allocations (condition i) above) and it is such that feasible allocations are not renegotiated (condition ii) above). The latter condition is made in order to separate the issue of infeasibility from the issue of pure renegotiation. ${ }^{3}$

If the reversion function can be chosen by the planner, under weak conditions any single valued SCR can be implemented as the next example shows.

Example 1. Assume that there is a state of the world, say $\omega^{\prime}$, in which the feasible set is larger than in any other state, i.e. $A(\omega) \subset A\left(\omega^{\prime}\right)$, any $\omega \neq \omega^{\prime}$. Then, any single-valued SCR such that $F\left(\omega^{\prime}\right) \in A\left(\omega^{\prime}\right) \backslash \bigcup_{\omega^{\prime} \neq \omega} A(\omega)$ can be implemented for some reversion function: Take a constant mechanism $g(m)=F\left(\omega^{\prime}\right), \forall m \in M$ and a reversion function $h\left(F\left(\omega^{\prime}\right), \omega\right) \equiv F(\omega), \forall \omega \in \Omega$. h fulfils the conditions for a reversion function since $F\left(\omega^{\prime}\right)$ is unfeasible at any state different than $\omega^{\prime}$ and by definition it provides the desired allocations. Notice that implementation occurs in dominant strategies.

The previous example suggests that in order to obtain meaningful results is better not to allow the designer of the mechanism to design the reversion function. This requirement is also intuitively agreeable because it seems reasonable there are aspects of the renegotiation that scape to the control of the mechanism designer.

To explain the main idea of the paper, consider the simplest possible example. Assume that at states of the world $\omega$ and $\omega^{\prime}$ the preference profile, say $R$, is the same.

\footnotetext{
${ }^{3} \mathrm{~A}$ tautological interpretation of the latter condition is that $A(\omega)$ is the set of allocations that are not renegotiated.
} 
Let $a, b$ and $c$ three allocations that are feasible at state $\omega$. Assume that $a P_{i} b P_{i} c$ for some agent $i$. In state $\omega^{\prime} a$ is not feasible and is renegotiated to $c$ and $b$ is feasible. So, even if the underlying preferences are the same in both states, player $i$ prefers $a$ to $b$ at state $\omega$ and $b$ to $a$ at $\omega^{\prime}$. To formalize and extend this idea we give the following definition.

Definition 2. Given $\omega \in \Omega$ and a reversion function $h$, the reversion of $R(\omega)$ on $A(\omega)$, denoted by $R^{h}(\omega)$ is

$$
a R_{i}^{h}(\omega) b \Leftrightarrow h(a, \omega) R_{i}(\omega) h(b, \omega), \forall a, b \in A, i \in I .
$$

With $L_{i}^{h}(a, \omega)=\left\{b \in A: h(a, \omega) R_{i}(\omega) h(b, \omega)\right\}$ we will denote the lower contour set of $a$ at $\omega$ with respect to $R^{h}(\omega)$.

Then, when the reversion function is $h$, we can interpret that agents' preferences are the reverted preferences, i.e. they only care about reverted allocations. Next definition is a straightforward adaptation of the standard notion of implementation in Nash equilibrium.

Definition 3. A social choice rule $F$ is h-implementable in Nash Equilibrium if there exists a game form $(M, g)$ such that for all $\omega \in \Omega$

$$
F(\omega)=h\left(N E\left(M, g, R^{h}(\omega)\right)\right)
$$

In words, $F$ is $h$-implementable in Nash equilibrium if and only if it is implementable in Nash equilibrium when for each $\omega \in \Omega$ the correspondent preference profile is $R^{h}(\omega)$. In other words, once we consider that agents' preferences are those induced by the reversion function, we can deal with $h$-implementation exactly in the same way as done in the classical implementation problem.

In the study of the restrictions that a state dependent feasible set imposes on implementation we concentrate on monotonicity (or Maskin-monotonicity). As observed 
by Jackson (2001), monotonicity is the most important obstacle to implementation in Nash equilibrium. For instance it is not generally satisfied by the Walrasian social choice rule. Monotonicity is, necessary, and almost sufficient condition for a SCR to be implementable in Nash equilibrium (see Maskin (1999) or Repullo (1987)). So it is the first condition to deal with.

A SCR satisfies monotonicity whenever an alternative is chosen at an state of the world and it rises in each agent's preference ranking at another state of the world, then it must be chosen also at this state. Now we restate the definition of monotonicity in terms of reverted preferences. Let $h$ be a reversion function.

Definition 4. A social choice rule $F$ is $h$-monotonic if for any $\omega, \omega^{\prime} \in \Omega$ and a such that $h(a, \omega) \in F(\omega)$ such that $L_{i}^{h}(a, \omega) \subset L_{i}^{h}\left(a, \omega^{\prime}\right)$ for all agents $i$ then $h\left(a, \omega^{\prime}\right) \in F\left(\omega^{\prime}\right)$.

The importance of $h$-monotonicity is highlighted by the following remark whose proof is an straightforward adaptation of an standard result and, therefore, is omitted:

Remark 1. If a social choice rule is h-implementable in Nash equilibrium, it is $h$ monotonic. Moreover in economic environments with $\# I>2$ if a social choice rule is $h$-monotonic, it is $h$-implementable in Nash equilibrium. ${ }^{4}$

\section{Non-Rewarding Reversion Functions: Basic Results}

In this section, we restrict our attention to a class of reversion functions where renegotiation is not advantageous for all players. We will call them Non-Rewarding. We will show that inside this class a particular renegotiation function -that we will call Generalized Severe- implements the maximal set of SCR. Then, we will characterize the SCR that can be implemented under generalized severe renegotiation functions.

\footnotetext{
${ }^{4} \mathrm{An}$ economic environment is one in which no two agents agree on the top allocation on their preference rankings.
} 
Let us start by defining the following class of reversion functions:

Definition 5. A reversion function is non-rewarding if for $a \in A(\omega)$ either there exists $i \in I$ and $c \in A\left(\omega^{\prime}\right)$ such that $a R_{i}(\omega) h(c, \omega)$ with $c P_{i}\left(\omega^{\prime}\right) h\left(a, \omega^{\prime}\right)$ or $L_{j}^{h}(a, \omega) \subset L_{j}^{h}\left(a, \omega^{\prime}\right)$ for all $j$.

Consider the case where the feasible set is constant and only preferences change. In this case the first condition in the definition postulates the existence of a pair of allocations for which there is a preference reversal. ${ }^{5}$ In the case where preferences are fixed and the feasible set varies, the idea is that when agents renegotiate something bad happens -delays, punishments engineered from the designer, etc.- and this what it causes the inversion of the ranking of $a$ and $c$ in the reverted preferences. The alternative in the definition considers the case where $a$ has improved in everybody ranking when passing from $\omega$ to $\omega^{\prime}$. It takes care of the case where $A\left(\omega^{\prime}\right) \subset A(\omega)$ because if $a \in A\left(\omega^{\prime}\right)$ no feasible reversal around $a$ can take place.

Consider now an specific reversion function which belongs to the class of nonrewarding ones. Suppose that should an infeasibility arise, players are redirected to what they consider to be the worst possible allocation. This reversion function resembles the assumption made in previous papers that agents do not choose unfeasible messages because the planner detects infeasibility and imposes a punishment in such a way that agents prefer any other feasible allocation to this punishment. However our interest in this particular renegotiation function arises from the fact it allows to find the maximal set of SCR that can be implemented under non-rewarding renegotiation (see Proposition 1 below).

Let $G \in A(\omega), \forall \omega \in \Omega$ be such that for all $i, a P_{i}(\omega) G$ with $a \neq G$ and $a \in A(\omega) . G$ will be called the "generalized punishment point". The reversion function with $h(a, \omega)=$

\footnotetext{
${ }^{5}$ This condition was emphsized by Maskin and Moore (1991): "The other problem that renegotiation poses is that interferes with "preference reversal" .
} 
$G$ if $a \notin A(\omega)$ will be called generalized severe and the induced preferences $R^{h}(\omega)$ will be called the saturation of $R(\omega)$ on $A(\omega)$ defined by the following properties.

For all $i \in I$ :

(1) If $a, b \in A(\omega)$ then $a R_{i}^{h}(\omega) b$ if and only if $a R_{i}(\omega) b$

(2) If $a \in A(\omega)$ and $b \notin A(\omega)$ then $a P_{i}^{h}(\omega) b$

(3) If $a, b \notin A(\omega)$ then $a I_{i}^{h}(\omega) b$

We show that generalized severe renegotiation implements the largest set of social choice rules among the class of non-rewarding reversion functions.

Proposition 1. Let $F$ be a SCR which is $h$-implementable in Nash Equilibrium with a non rewarding reversion function. Then $F$ is implementable in Nash Equilibrium with a generalized severe reversion function.

Proof Let $(M, g)$ implementing $F$ with reversion function $h$.

Let $a \in F(\omega)$. Let $m(\omega, a)$ be a Nash equilibrium of $\left\{M, g ; R^{h}(\omega)\right\}$ such that $g(m(\omega, a))=a$.

Let $B_{i}(\omega, a)=g\left(M_{i} \times\left\{m_{-i}(\omega, a)\right\}\right)$ be the attainable set of $i$.

Set $B_{i}^{h}(\omega, a)=\bigcup_{\omega^{\prime} \in \Omega}\left\{c \in A\left(\omega^{\prime}\right): a R_{i}(\omega) h(c, \omega), c P_{i}\left(\omega^{\prime}\right) h\left(a, \omega^{\prime}\right)\right.$ if for some $b \in$ $\left.B_{i}(\omega, a), a R_{i}(\omega) h(b, \omega), h\left(b, \omega^{\prime}\right) P_{i}\left(\omega^{\prime}\right) h\left(a, \omega^{\prime}\right)\right\}$

Set $B^{h}=\operatorname{Im} g \cup\left(\bigcup_{\substack{\omega, \omega^{\prime} \in \Omega \\ i \in I}}\left\{c \in A\left(\omega^{\prime}\right): a R_{i}(\omega) h(c, \omega), a P_{i}\left(\omega^{\prime}\right) h\left(c, \omega^{\prime}\right)\right.\right.$ if for some $\left.\left.b \in B_{i}(\omega, a), a R_{i}(\omega) h(b, \omega), h\left(b, \omega^{\prime}\right) P_{i}\left(\omega^{\prime}\right) h\left(a, \omega^{\prime}\right)\right\}\right)$.

Observe that for all $\omega \in \Omega, a \in F(\omega)$, and $i, B_{i}^{h}(\omega, a) \subset B^{h}$. Thus:

$-B_{i}^{h}(\omega, a) \cap A(\omega) \subset B_{i}(\omega, a) \cap A(\omega)$ for all $\omega$ $-a R_{i}^{h}(\omega) x$ for all $x \in B_{i}(\omega, a)$ and for all $i \in I$ -if $a R_{i}\left(\omega^{\prime}\right) x$ for all $i \in I, x \in B_{i}^{h}(\omega, a) \cap A\left(\omega^{\prime}\right)$ then $a \in F\left(\omega^{\prime}\right)$. Otherwise there would exist $j \in I$ and $b \in B_{j}(\omega, a)$ such that $h\left(b, \omega^{\prime}\right) P_{i}\left(\omega^{\prime}\right) h\left(a, \omega^{\prime}\right)$. $h$ is nonrewarding so there exists $c \in A\left(\omega^{\prime}\right)$ such that $a R_{j}(\omega) h(c, \omega), c P_{j}\left(\omega^{\prime}\right) h\left(a, \omega^{\prime}\right)$. By definition such a $c$ belongs to $B_{j}^{h}(\omega, a)$. 
Using the assumption that $h$ is non-rewarding we can prove, exactly as above: -if $b \in B_{i}^{h}(\omega, a)$ for some $i \in I$ is such that for some $\omega^{\prime} \in \Omega, b R_{i}\left(\omega^{\prime}\right) x$ for all $x \in B_{i}^{h}(\omega, a) \cap A\left(\omega^{\prime}\right)$, and if $b R_{j}^{h}\left(\omega^{\prime}\right) x$ for all $x \in B^{h} \cap A\left(\omega^{\prime}\right)$ for each $j \neq i$ then $b \in F\left(\omega^{\prime}\right)$.

-if $b \in B^{h}$ is such that for some $\omega^{\prime} \in \Omega, b R_{i}^{h}\left(\omega^{\prime}\right) x \forall x \in B$ fore all $i$, then $b \in F\left(\omega^{\prime}\right)$ For all $i$ set $M_{i}^{\prime}=\{(\omega, a) ; a \in F(\omega)\} \times B^{h} \times \mathbf{N}$, where $\mathbf{N}$ is the set of integers. Let $M^{\prime}=\prod_{1}^{N} M_{i}^{\prime}$, and $g^{\prime}: M^{\prime} \longrightarrow A$ such that:

a) $g^{\prime}(m)=a$ if $m_{i}=(\omega, a, b, n) \forall i$.

b) If there exists a unique $i$ such that for all $j \neq i m_{j}=(\omega, a, b, n)$ and $m_{i}=$ $\left(\omega_{i}, a_{i}, b_{i}, n_{i}\right)$ is such that $(\omega, a, b, n) \neq\left(\omega_{i}, a_{i}, b_{i}, n_{i}\right)$ then set $g^{\prime}(m)=b$ if $b_{i} \in$ $B_{i}^{h}(\omega, a)$, otherwise set $g^{\prime}(m)=a$.

c) Otherwise set $g^{\prime}(m)=b_{i}$ where $i=\min \arg \max _{j}\left\{n_{j} ; m_{j}=\left(\omega_{j}, a_{j}, b_{j}, n_{j}\right)\right\}$.

This is the canonical mechanism in Nash implementation. It is immediate to prove that $\left(M^{\prime}, g^{\prime}\right)$ implements $F$ by generalized severe punishment.

In words, generalized severe renegotiation implements any SCR implementable with non-rewarding reversion functions. ${ }^{6}, 7$ The rest of this section will be devoted to study

\footnotetext{
${ }^{6}$ Proposition 1 can be proved under the following assumption that generalizes that of a non-rewarding reversion function: whenever there exists $i \in I$ with $a R_{i}(\omega) h(b, \omega)$ and $h\left(b, \omega^{\prime}\right) P_{i}\left(\omega^{\prime}\right) h\left(a, \omega^{\prime}\right) a \in A(\omega)$ then there exists $j$ and $c \in A\left(\omega^{\prime}\right)$ such that: i) $a R_{j}(\omega) h(b, \omega)$ and $h\left(b, \omega^{\prime}\right) P_{j}\left(\omega^{\prime}\right) h\left(a, \omega^{\prime}\right)$ and ii) $a R_{j}(\omega) h(c, \omega)$ with $c P_{j}\left(\omega^{\prime}\right) h\left(a, \omega^{\prime}\right)$. This says that at least one agent suffers as a consequence of unfeasibility in a way that could have been made through a feasible allocation. For instance, the agent who is deemed responsible for the unfeasibility is punished and there is an agent who does not get the bundle she consumed at the other state but what she consumes is feasible.

${ }^{7}$ The assumption of no rewarding is necessary for Proposition1 to hold. Let $\Omega=\left\{\omega, \omega^{\prime}\right\}, A(\omega)=$ $\{a, b, c, G\}$ and $A\left(\omega^{\prime}\right)=\{a, b, G\}$. Let $n=2$ and $R_{i}(\omega)=R_{i}\left(\omega^{\prime}\right)=R$ for $i=1,2$ where $b P a P c$. Let $F(\omega)=a$ and $F\left(\omega^{\prime}\right)=b$. Let $h\left(c, \omega^{\prime}\right)=b . h$ does not satisfies the non rewarding assumption at $c$. $F$ is $h$-implementable in NE by the simple mechanism which leaves agent 1 (or 2) choose among $a$ and $c$. But it cannot be implemented by severe generalized punishment because $F($ ) it is not monotonic with respect to saturated preferences.
} 
the former. According to Remark 1 this leads us to study $h$-monotonicity under saturated preferences.

We now introduce two properties that are necessary and sufficient for $h$-monotonicity under generalized severe renegotiation.

Definition 6. $F$ satisfies weak unanimity if for all $\omega, \omega^{\prime} \in \Omega$ such that $A\left(\omega^{\prime}\right) \subset A(\omega)$ and for all $a \in A(\omega) \backslash A\left(\omega^{\prime}\right)$ such that $L_{i}(a, \omega) \cap A\left(\omega^{\prime}\right) \subset L_{i}\left(a, \omega^{\prime}\right)$ for all $i \in I, a \notin F(\omega)$.

When preferences are fixed, Weak Unanimity says that if all alternatives available at $\omega^{\prime}$ are also available at $\omega$, the SCR will not select at $\omega$ an alternative which is available at $\omega$ but not at $\omega^{\prime}$ if all players prefer any allocation available at $\omega^{\prime}$ to it. If this condition is not satisfied, when the actual economy is $A(\omega)$ all agents have incentives to underrepresent the economy and implement the decision intended for state $\omega^{\prime}$. Weak unanimity is equivalent to the following condition: if $A\left(\omega^{\prime}\right) \subset A(\omega)$ and $a \in F(\omega) \backslash A\left(\omega^{\prime}\right)$ then there exists $b \in A\left(\omega^{\prime}\right), b \neq G$ such that $a R_{i} b$ for some $i \in I$.

Definition 7. F satisfies generalized contraction consistency $(G C C)$ if, for $\omega, \omega^{\prime} \in \Omega$, and for $a \in F(\omega) \cap A\left(\omega^{\prime}\right)$ such that $L_{i}(a, \omega) \cap A(\omega) \cap A\left(\omega^{\prime}\right) \subset L_{i}\left(a, \omega^{\prime}\right)$ and $A\left(\omega^{\prime}\right) \backslash A(\omega) \subset$ $L_{i}\left(a, \omega^{\prime}\right)$.for all $i \in I, a \in F\left(\omega^{\prime}\right)$.

In the case preferences are fixed and $A\left(\omega^{\prime}\right) \subset A(\omega), A\left(\omega^{\prime}\right) \backslash A(\omega)=\varnothing \subset L_{i}(a, \omega)$ for all $i$. In such a case GCC prescribes to choose at state $\omega^{\prime}$ any feasible allocation we have chosen at state $\omega$. Thus GCC is a weak version of Nash Independence of Irrelevant Alternatives (see Roemer [1996], p. 55). GCC says that if $a$ is selected at state $\omega$, and is feasible also at $\omega^{\prime}$ and no better alternatives are available in $A\left(\omega^{\prime}\right) \backslash A(\omega)$, then $a$ must be selected also at state $\omega^{\prime}$.

Proposition 2. A feasible $F$ is monotonic under generalized severe punishment if and only if it satisfies generalized contraction consistency and weak unanimity. 
Proof Let $h$ to denote the generalized severe punishment reversion function.

We begin by proving the necessity of weak unanimity and GCC. Let $F$ be $h$ monotonic.

Then $F$ must satisfy weak unanimity. Let $\omega, \omega^{\prime} \in \Omega$, let $A\left(\omega^{\prime}\right) \subset A(\omega)$ and let $a \in A(\omega) \backslash A\left(\omega^{\prime}\right)$ such that $L_{i}(a, \omega) \cap A\left(\omega^{\prime}\right) \subset L_{i}\left(a, \omega^{\prime}\right)$ and $i \in I$. By contradiction, let $a \in F(\omega)$. Then $L_{i}^{h}(a, \omega)=\left(L_{i}(a, \omega) \cap A(\omega)\right) \cup A \backslash A(\omega)=\left(L_{i}(a, \omega) \cap A\left(\omega^{\prime}\right)\right) \cup$ $\left(L_{i}(a, \omega) \cap A(\omega) \backslash A\left(\omega^{\prime}\right)\right) \cup A \backslash A(\omega) \subset\left(L_{i}\left(a, \omega^{\prime}\right) \cap A\left(\omega^{\prime}\right)\right) \cup A \backslash A\left(\omega^{\prime}\right)=L_{i}^{h}\left(a, \omega^{\prime}\right)$ for all $i \in I$. Then $h$-monotonicity implies that $a \in F\left(\omega^{\prime}\right)$, which is a contradiction as $F\left(\omega^{\prime}\right) \subset A\left(\omega^{\prime}\right)$.

Now we consider GCC. Let $a \in F(\omega) \cap A\left(\omega^{\prime}\right)$ such that $L_{i}(a, \omega) \cap A(\omega) \cap A\left(\omega^{\prime}\right) \subset$ $L_{i}\left(a, \omega^{\prime}\right)$ and $A\left(\omega^{\prime}\right) \backslash A(\omega) \subset L_{i}\left(a, \omega^{\prime}\right)$ for all $i \in I$.

$$
\begin{gathered}
L_{i}^{h}(a, \omega)=\left(L_{i}(a, \omega) \cap A(\omega) \cap A\left(\omega^{\prime}\right)\right) \cup\left(L_{i}(a, \omega) \cap A(\omega) \backslash A\left(\omega^{\prime}\right)\right) \cup \\
\left(A\left(\omega^{\prime}\right) \backslash A(\omega)\right) \cup\left((A \backslash A(\omega)) \backslash A\left(\omega^{\prime}\right)\right) \subset\left(L_{i}\left(a, \omega^{\prime}\right) \cap A(\omega) \cap A\left(\omega^{\prime}\right)\right) \cup \\
\left(L_{i}\left(a, \omega^{\prime}\right) \cap A\left(\omega^{\prime}\right)\right) \cup A \backslash A\left(\omega^{\prime}\right)=L_{i}^{h}\left(a, \omega^{\prime}\right) \text {. h-monotonicity implies } a \in F\left(\omega^{\prime}\right) .
\end{gathered}
$$

We next show the sufficiency of weak unanimity and GCC for $F$ to be $h$-monotonic.

Let $\omega, \omega^{\prime} \in \Omega$, and let $a \in F(\omega)$ such that $L_{i}^{h}(a, \omega) \subset L_{i}^{h}\left(a, \omega^{\prime}\right)$ for all $i$. Consider the following three cases.

i) $A(\omega) \cap A\left(\omega^{\prime}\right)=\{G\}$

ii). $A(\omega) \cap A\left(\omega^{\prime}\right) \neq\{G\}$ and $a \notin A\left(\omega^{\prime}\right)$

iii) $A(\omega) \cap A\left(\omega^{\prime}\right) \neq\{G\}$ and $a \in A\left(\omega^{\prime}\right)$

i) It is not possible. In such a case or no $i \in I L_{i}^{h}(a, \omega) \subset L_{i}^{h}\left(a, \omega^{\prime}\right)$, since, from the definition of saturated preferences it follows that $a P_{i}^{h}(\omega) b$ and $b P_{i}^{h}\left(\omega^{\prime}\right) a$ for all $b \in A\left(\omega^{\prime}\right)$. ii) It must be the case that $A\left(\omega^{\prime}\right) \subset A(\omega)$. Otherwise from the definition of saturated preferences for all $b \in A\left(\omega^{\prime}\right) \backslash A(\omega): a P_{i}^{h}(\omega) b$ and $b P_{i}^{h}\left(\omega^{\prime}\right) a$ for all $i$. Then we must have 
$L_{i}(a, \omega) \cap A\left(\omega^{\prime}\right) \subset L_{i}\left(a, \omega^{\prime}\right)$ for all $i \in I$ Otherwise for some $i \in I, . b \in A\left(\omega^{\prime}\right): a R_{i}(\omega) b$ and $b P_{i}\left(\omega^{\prime}\right) a$. But from weak unanimity it would follow that $a \notin F(\omega)$, a contradiction. iii) It must be the case that $L_{i}(a, \omega) \cap A(\omega) \cap A\left(\omega^{\prime}\right) \subset L_{i}\left(a, \omega^{\prime}\right)$ and

$A\left(\omega^{\prime}\right) \backslash A(\omega) \subset L_{i}\left(a, \omega^{\prime}\right)$ for all $i \in I$. Otherwise either there exists $b \in A(\omega) \cap A\left(\omega^{\prime}\right)$ such that $a R_{i}(\omega) b$ and $b P_{i}\left(\omega^{\prime}\right) a$ for some $i \in I$ or there exists $b \in A\left(\omega^{\prime}\right) \backslash A(\omega)$ such that $a P_{i}^{h}(\omega) b$ and $b P_{i}\left(\omega^{\prime}\right) a$ for some $i \in I$. Then GCC implies that $a \in F\left(\omega^{\prime}\right)$.

So $F$ is monotonic.

\section{Non-Rewarding Reversion Functions: Applications}

In this section we apply the findings of previous sections to several set ups. We begin by considering pure exchange economies. In such environment with more than two agents, $h$-monotonicity is necessary and sufficient for $F$ to be $h$-implementable in Nash equilibrium.

\subsection{Consumption as allocation}

Let $X_{i}=\mathbf{R}_{+}^{K}$ be $i$ 's consumption set. We assume that agents' preferences and consumption sets do not vary but endowments do. Let $u_{i}$ be an increasing and concave utility function representing agent $i$ 's preferences. Let $\Omega_{i} \subset \mathbf{R}_{+}^{K}$ be the set of agent $i$ 's possible endowments. For $\omega=\left(\omega_{1}, \ldots, \omega_{n}\right) \in \Omega$ set $\bar{\omega}=\sum_{i=1}^{n} \omega_{i}$. Set $A(\omega)=\left\{x \in \mathbf{R}_{+}^{K n}: \sum x_{i}=\bar{\omega}\right\} \cup\{G\}$ the set of fully balanced consumption bundles plus the generalized punishment point. ${ }^{8}$ Here feasibility refers just to balancedness. A representation for saturated preferences is:

$$
\begin{aligned}
u_{i}^{w}(x) & =u_{i}\left(x_{i}\right), x \in A(\omega) \backslash\{G\} \\
u_{i}^{w}(G) & =u_{i}(0)-\varepsilon, \varepsilon>0 .
\end{aligned}
$$

\footnotetext{
${ }^{8}$ The same result holds if we consider the feasible set as $A(\omega)=\left\{x \in \mathbf{R}_{+}^{K n}: \sum x_{i} \leq \bar{\omega}\right\} \cup\{G\}$.
} 
Observe that $A\left(\omega^{\prime}\right)=A(\omega)$ if and only if $\bar{\omega}^{\prime}=\bar{\omega}$. Otherwise $A\left(\omega^{\prime}\right) \cap A(\omega)=\varnothing$.

Weak unanimity is satisfied because if $A\left(\omega^{\prime}\right) \subset A(\omega)$ then $\bar{\omega}^{\prime}=\bar{\omega}$ and $A\left(\omega^{\prime}\right)=A(\omega)$. Then for all $x \in F(\omega), x \in A\left(\omega^{\prime}\right)$

GCC is binding only when $\bar{\omega}^{\prime}=\bar{\omega}$, too. In such a case GCC prescribes that $F(\omega)=$ $F\left(\omega^{\prime}\right)$ because agents' lower contour sets do no vary. It follows that a SCR can be implemented in Nash equilibrium if and only if it can be written as function of the aggregate endowment $F(\omega)=F(\bar{\omega})$. This is a strong condition that is not satisfied by any SCR picking up individually rational allocations. However this condition is satisfied by SCR which embody just an idea of fairness, like envy-free allocations.

\subsection{Net transfers as allocations}

We assume preferences on consumptions bundles are known and satisfy the same conditions as above. We consider that the planner can only transfer goods among players. Then the allocation set contains the set of the balanced net transfers and the generalized punishment point, $A=\left\{x \in R^{K \times n}: \sum_{s=1}^{K} x_{s}=0\right\} \cup\{G\}$. For all $\omega \in \Omega$ the feasible set is $A(\omega)=\left\{x \in A: x_{i}+\omega_{i} \geq 0\right.$ for $\left.i=1, \ldots, n\right\} \cup\{G\}$. Then $A\left(\omega^{\prime}\right) \subset A(\omega)$ if and only if $\omega^{\prime} \leq \omega$.

In order to describe preferences on net transfers we need to make them state dependent because the utility agent $i$ gets from transfer $x_{i}$ when her endowment is $\omega_{i}$ is $u_{i}\left(x_{i}+\omega_{i}\right)$. For each $\omega$ and for all $x \in A(\omega)$ for all $i$ set $u_{i}(x, \omega) \equiv u_{i}\left(x_{i}+\omega_{i}\right)$.

Saturated preferences can be represented by the following utility functions.

$$
\begin{aligned}
u_{i}^{\omega}(x) & =u_{i}(x, \omega) x \in A(\omega) \backslash\{G\} \\
u_{i}^{\omega}(G) & =u_{i}(0)-\varepsilon, \varepsilon>0 .
\end{aligned}
$$

Let us consider first weak unanimity. It is easily seen that it suffices to consider only endowments $\omega, \omega^{\prime}$ such that $\omega^{\prime} \leq \omega$. Then weak unanimity amounts to the following condition: 
Condition $\alpha$ : For all $\omega, \omega^{\prime} \in \Omega$ such that $\omega^{\prime} \leq \omega$, if $a \in F(\omega) \backslash A\left(\omega^{\prime}\right)$ there exists $i$ such that $u_{i}\left(\omega_{i}+a_{i}\right) \geq u_{i}\left(\omega_{i}-\omega_{i}^{\prime}\right)$.

Observe that if $\left(0, \omega_{i}\right) \subset \Omega_{i}$ for all $i$ then Condition $\alpha$ requires simply the SCR to be individually rational for at least one agent. It is a very weak requirement and it is obviously satisfied by many SCR, e.g., any Pareto efficient or any individually rational SCR.

Stronger requirements are imposed by GCC. Also in this case it suffices to consider only endowments $\omega, \omega^{\prime} \in \Omega$ such that $\omega^{\prime} \leq \omega$. GCC is satisfied if and only if the following condition holds:

Condition $\beta$. For all $\omega, \omega^{\prime} \in \Omega$ such that $\omega^{\prime} \leq \omega$, if $a \in F(\omega) \cap A\left(\omega^{\prime}\right)$ and $a \notin F\left(\omega^{\prime}\right)$ there exists $i$ and $x \in A\left(\omega^{\prime}\right)$ such that

$$
\begin{aligned}
& u_{i}\left(\omega_{i}+a_{i}\right) \geq u_{i}\left(\omega_{i}+x_{i}\right) \\
& u_{i}\left(\omega_{i}^{\prime}+a_{i}\right)<u_{i}\left(\omega_{i}^{\prime}+x_{i}\right)
\end{aligned}
$$

Let us now analyze the possibility of implementing the Constrained Walrasian Social Choice Rule (CWC) by generalized severe punishment. $a$ is a Constrained Walrasian Allocation (CWA) at $\omega$ iff there exists $p \in \mathbf{R}_{+}^{K}$ such that, for all $i=1, \ldots, n$ $a \in \arg \max \left\{u_{i}\left(\omega_{i}+x_{i}\right): p x_{i} \leq 0, x \in A(\omega)\right\} . p$ is said to be an equilibrium price supporting $a$ at $\omega$. Let $C W C(\omega)$ denote the set of CWA at $\omega$.

Proposition 3. Let preferences be increasing, continuous and strictly convex and let $\Omega_{i}=\left(0, \bar{\omega}_{i}\right)$ for all $i$ for some $\bar{\omega}_{i} \in(0, \infty)$. Then the Constrained Walrasian social choice rule is implementable in Nash Equilibrium by generalized severe punishment.

Proof Under our assumptions $C W C(\omega)$ is not empty for all $\omega \in \Omega$. To prove the claim it suffices to show that $C W C$ satisfies Condition $\beta$. Let $\omega^{\prime} \leq \omega, a \in$ $C W C(\omega) \cap A\left(\omega^{\prime}\right)$ and $a \notin C W C\left(\omega^{\prime}\right)$. Let $p$ an equilibrium price at $\omega$. Then there exists $x \in A\left(\omega^{\prime}\right)$ with $u_{i}\left(\omega^{\prime}+x_{i}\right)>u_{i}\left(\omega^{\prime}+a_{i}\right)$ and $p x_{i} \leq 0$ some $i . A\left(\omega^{\prime}\right) \subset A(\omega)$ 
so $x \in\left\{p x_{i} \leq 0, x \in A(\omega)\right\}$. From the definition of $C W C$ it follows $u_{i}\left(\omega_{i}+a_{i}\right) \geq$ $u_{i}\left(\omega_{i}+x_{i}\right)$. Then $C W C$ satisfies Condition $\beta .^{9}$

Let us compare our results with Hong (1998). She showed that a SCR is implementable by a collection of state dependent mechanisms if and only if the following condition is satisfied

$$
u_{i}\left(\omega_{i}+f_{i}(\omega)\right) \geq u_{i}\left(\omega_{i}-\omega_{i}^{\prime}\right) \text { for all } i
$$

Our Condition $(\alpha)$ is weaker than condition (H): If $x \in A\left(\omega^{\prime}\right)$ then $u_{i}\left(\omega_{i}+x_{i}\right) \geq u_{i}\left(\omega_{i}-\right.$ $\left.\omega_{i}^{\prime}\right)$ for all $i$ as all $u_{i}$ are increasing. Then if $f(\omega) \in A\left(\omega^{\prime}\right), u_{i}\left(\omega_{i}+f_{i}(\omega)\right) \geq u_{i}\left(\omega_{i}-\omega_{i}^{\prime}\right)$ for all $i$. So for $\omega, \omega^{\prime}$ with $f(\omega) \in A\left(\omega^{\prime}\right)$ condition (H) holds. Notice that our condition depends on the fact that each agent cannot simply retain part of her endowment, but it has to do it compatible with other agents' messages.

But our Condition $(\beta)$ is not implied by Condition $(\mathrm{H})$. Assume for instance that $f(\omega) \in A\left(\omega^{\prime}\right)$ then $(\mathrm{H})$ imposes no restrictions on $f\left(\omega^{\prime}\right)$. If the translations by $\omega-\omega^{\prime}$ of all agents' indifference curves through $\omega^{\prime}+f(\omega)$ are strictly above all agents' indifferences curves through $\omega+f(\omega)$ then condition $(\beta)$ implies $f(\omega)=f\left(\omega^{\prime}\right)$. Formally if for all $y \in\left\{y: u_{i}\left(\omega_{i}^{\prime}+f_{i}(\omega)\right)\right)=u_{i}\left(y_{i}\right)$ for all $\left.i\right\}$ we have $u_{i}\left(y_{i}+\omega_{i}-\omega_{i}^{\prime}\right)>u_{i}\left(\omega_{i}+f_{i}(\omega)\right)$ for all $i$, condition $(\beta)$ imposes that $f(\omega)=f\left(\omega^{\prime}\right)$.

The difference between our conditions and Hong's is explained by the fact that her goal is to design one feasible mechanism $(M(\omega), g(\omega))$ for each possible endowments $\omega$, in a way such that the larger the feasible set, the larger the message space. Two of her assumptions make our approaches different:

i) Hong assumes that players can not exaggerate their endowment and that they can be punished by the message they send not only for the allocation they intend to obtain, if

\footnotetext{
${ }^{9}$ The Walrasian Correspondence $W C$ defined by $W C(\omega)=\arg \max \left\{u_{i}\left(\omega_{i}+x_{i}\right): p x_{i} \leq 0\right\}$ is not implementable in Nash Equilibrium by generalized severe punishment. An example is available from the authors under request but intuitively is clear that in our case preferences vary so we are back to the classical framework where such a problem is well known.
} 
such an allocation is not feasible.

ii) Hong gives to each player the power of retaining part of her endowments. In our framework we assume that players can collectively cheat the planner through the mechanism by asking a feasible allocation in which some agents retain a part of her endowment.

\subsection{Bargaining with unknown utility possibility set}

We now consider the non-cooperative implementation of cooperative solution concepts (Dagan and Serrano [1998]).

A bargaining problem is a pair $(U, v)$ where $U \subset \mathbf{R}_{+}^{n}$ is the utility possibility set and $v \in U$ is the disagreement point. We assume that $U$ is convex, closed, with a non empty interior and comprehensive (i.e. $u \in U$ and $u^{\prime} \leq u, u^{\prime} \in \mathbf{R}_{+}^{n}$ implies $u^{\prime} \in U$ ). For each bargaining problem, $(U, v)$ let $U_{v}=\{u \in U: u \geq v\}$ be bounded. The Nash Bargaining Solution (NBS) is defined as $N B S(U, v)=\arg \max _{u \in U_{v}} \prod_{i=1}^{n}\left(u_{i}-v_{i}\right)$. It is completely characterized by the following properties: strong efficiency, individual rationality, scale covariance, symmetry and independence of irrelevant alternatives. Let $N B S(U, v)_{i}$ be the utility received by $i$.

We consider here non rewarding renegotiation function more suited to the situation. We say that a reversion function is Not Severe if for all $a \in A$ and $\omega \in \Omega h(a, \omega) \neq G$.

We consider $U_{v}$ as feasible set of $(U, v)$ and we assume that unfeasible allocations are renegotiated to the disagreement point. Let $h$ to denote such renegotiation function. Clearly $h$ is Non Rewarding. Agent $i$ 's reverted preferences at $(U, v)$ are described by

$$
\begin{gathered}
\left.u_{i}^{h}(u(U, v))\right)=u_{i} \text { if } u \in U_{v} \\
u_{i}^{h}(u(U, v))=v_{i} \text { otherwise. }^{10}
\end{gathered}
$$

\footnotetext{
${ }^{10}$ Naeve (1999) defines preferences on the utility set as they was the reverted preferences. with the unique differences that utility vector in $U \backslash U_{v}$ are not renegotiated. A renegotiation consistent with this view would not alter our results.
} 
If the disagreement point is not known by the planner, NBS fails to satisfy GCC and, according to Proposition 1, is not implementable in NE by any non-rewarding renegotiation function. ${ }^{11}$ Let $n=2$ and let $U=\left\{x \in \mathbf{R}_{+}^{2}: x_{1}^{2}+x_{2}^{2} \leq 1\right\}$. Let $v=$ $(0,0)$ and let $v^{\prime}=\left(\left(\frac{1}{2}\right)^{\frac{1}{2}}, 0\right)$. Then $N B S(U, v)=\left(\left(\frac{1}{2}\right)^{\frac{1}{2}},\left(\frac{1}{2}\right)^{\frac{1}{2}}\right) \in U_{v^{\prime}} \subset U_{v}$ but $N B S(U, v) \neq N B S\left(U, v^{\prime}\right)$.

The Kalai-Smorodinski solution does not satisfy GCC even with fixed disagreement point. Then Proposition 1 implies that it cannot be implemented in NE by any non rewarding renegotiation function.

Instead, when the disagreement point is known the NBS satisfies both GCC and weak unanimity as the reader can easily check. However Proposition 2 cannot be used to conclude that the NBS is implementable by generalized severe punishment because Maskin Theorem requires at least three agents. ${ }^{12}$

We proof the result directly by using the characterizations by Moore and Repullo (1990).

\footnotetext{
${ }^{11}$ This result agrees with the findings of Serrano (1997). A different interpretation of preferences on the utility possibility set may lead to more permissive results. One can interpret them as they were a measure of agents' satisfaction with respect to the disagreement point. A representation consistent with this view is $u_{i}(u,(U, v))=u_{i}-v_{i}$. Then the preferences that $h$ induces in this case are

$$
\begin{aligned}
& u_{i}^{h}(u,(U, v))=u_{i}-v_{i} \text { if } u \in U_{v} \\
& u_{i}^{h}(u,(U, v))=0 \text { otherwise }
\end{aligned}
$$

Observe that $u_{i}^{h}(u,(U, v))=u_{i}^{h}(u-v,(U-v, 0))$. The reader can easily check that from the translation invariance property of the $N B S$ the analysis of the problem with unknown endowments amounts to the previous situation with the endowment fixed and known at 0 . In this case applying Proposition 4 below yields a positive result.

${ }^{12} \mathrm{NBS}$ does not satisfy no-veto power either. As an example consider $U=$ $\left\{x \in \mathbf{R}_{+}^{3}: \max \left\{x_{1}, x_{2}\right\} \leq 1, \max \left\{x_{1}+x_{3}, x_{2}+x_{3}\right\} \leq 1\right\}$ and let $v=(0,0,0)$. Agent 1 and agent 2 prefer $u=(1,1,0) \in U$ to any other allocation, under saturated preferences but $N B S(U, v)=\left(\frac{2}{3}, \frac{2}{3}, \frac{1}{3}\right)$.
} 
Proposition 4. Let $n \geq 2$. The Nash Bargaining Solution is $h$-implementable in Nash Equilibrium with a Non-Severe $h$ if the disagreement point $v$ is known.

Proof Let $x=N B S(U, v)$. Let $i \in I$ and let $\left(U^{\prime}, v\right)$ be a bargaining problem. Let $u \in L_{i}^{h}(x,(U, v))$ such that, at $\left(U^{\prime}, v\right)$ and with reverted preferences $u$ is maximal for $i$ in $L_{i}^{h}\left(x,\left(U^{\prime}, v\right)\right)$ and $u$ is maximal in $\mathbf{R}_{+}^{n}$ for all agents different from $i$. We first prove that $u=N B S\left(U^{\prime}, v\right)$. Observe that it must be the case that $u$ is feasible at $U^{\prime}$ otherwise all agents different from $i$ would prefer some point in the interior of $U_{v}^{\prime}$ and that $u_{j}=\max \left\{u_{j}^{\prime}: u^{\prime}=\left(u_{j}^{\prime}, u_{-j}^{\prime}\right) \in U_{v}^{\prime}\right\}$ for all $j \neq i$. In particular $u$ lies on the boundary of $U^{\prime}$. If $u \neq N B S\left(U^{\prime}, v\right)$ then $N B S\left(U^{\prime}, v\right)_{i}>$ $u_{i}$. If $N B S\left(U^{\prime}, v\right) \notin U_{v} u$ is not maximal in $L_{i}^{h}(x,(U, v))$ for $i$ when preference are reverted at $\left(U^{\prime}, v\right)$, a contradiction. Finally consider the case $N B S\left(U^{\prime}, v\right) \in$ $U_{v} . N B S\left(U^{\prime}, v\right) \neq N B S(U, v)$ and $N B S(U, v) \notin U_{v^{\prime}}^{\prime}$ otherwise $u$ would not be maximal in $L_{i}^{h}(x,(U, v))$ for $i$ under reverted preferences. Consider the segment joining $N B S\left(U^{\prime}, v\right)$ and $u$. Such a segment lies in $U_{v}^{\prime}$ because $U_{v}^{\prime}$ is convex and it intersects $\left\{u^{\prime} \in U_{v}: N B S(U, v)_{i} \geq u_{i}^{\prime}\right\}$ because $U_{v}$ is convex and $N B S(U, v) \notin$ $U_{v}^{\prime}$. All along the segment the coordinate $i$ increases from $u_{i}^{\prime}$ to $N B S\left(U^{\prime}, v\right)_{i}$. Then there exists a point in $\left\{u^{\prime} \in U_{v}: N B S(U, v)_{i} \geq u_{i}^{\prime}\right\}$ which has the $i$-th coordinate strictly greater than $u_{i}$, a contradiction. Let $u$ be maximal in $R_{+}^{n}$ for all agents when preferences reverted at $\left(U^{\prime}, v\right)$ then $u_{j}=\max \left\{u_{j}^{\prime}: u^{\prime}=\left(u_{j}^{\prime}, u_{-j}^{\prime}\right) \in U_{v}^{\prime}\right\}$ for all $j$. From efficiency it follows that $u=N B S\left(U^{\prime}, v\right)$.

NBS satisfies Individual Rationality, Pareto efficiency and GCC, too. Then, when $n \geq 3$ the family of sets $\left\{L^{h}(x,(U, v))\right\}_{x=N B S(U, v)}$ satisfies condition $\mu$ in Moore and Repullo (1990). When $n=2$ it satisfies condition $\mu 1$ in the same paper, because of the disagreement point. Then the application of Theorems 1 and 2 there, respectively lead to the claim. 


\subsection{Taxation}

A taxation problem, is a pair $(x, T) \in R_{+}^{n} \times R_{+}$where $x$ is the vector of taxable incomes and $T$ is the total amount to be collected such that $\sum_{i=1}^{n} x_{i} \geq T$ (Dagan et alia [1999]). A tax allocation $t$ is a vector of $R_{+}^{n}$ and it is feasible for the taxation problem $(x, T)$ if $t \leq x$ and $\sum_{i=1}^{n} t_{i}=T$. A taxation method is a function $f$ which associates to each taxation problem a tax allocation. We assume that the planner knows the amount to be collected, $T$ but she does not know the taxable vector $x$. Let $S^{n}(T)=\left\{t \in R_{+}^{n}: \sum_{i=1}^{n} t_{i}=T\right\}$ be the set of tax allocations that collect $T$. Let $\Omega^{n}(T)=\left\{x \in R_{+}^{n}: \sum_{i=1}^{n} x_{i} \geq T\right\}$ be the set of the states of the world. Let $T^{n}(x)=T^{n}(x, T)=\left\{t \in R_{+}^{n}: 0 \leq t \leq x, \sum_{i=1}^{n} t_{i}=T\right\}$ be the tax allocations that are feasible at $x$. Each agent's preferences only depend on her after tax income and are strictly increasing. Then we can assume $u_{i}(t, x)=x_{i}-t_{i}$ for each $x \in \Omega^{n}(T)$ and for each $t \in T^{n}(x, T)$. Assume that only income exaggeration can be detected. In such a case no punishment is administrated. Then the reversion function is non severe. Observe that $h$ satisfies the hypothesis of Proposition ??. Then if a taxation method feasible $f$ is $h$ - implementable in NE it is implementable under non-rewarding reversion function.

Let $\sigma: I \rightarrow I$ be a permutation or ranking on the agents. Set $(i)=\sigma^{-1}(i)$. Set $f^{(\sigma)}$ to be the following feasible taxation method.

$$
\begin{aligned}
& f_{(1)}^{\sigma}(x)=\min \left\{t_{(1)}: t \in T^{n}(x, T)\right\} \\
& f_{(j)}^{\sigma}(x)=\min \left\{t_{(j)}: t \in T^{n-j+1}\left(x_{-\{(1), \ldots,(j-1)\}}, T-\sum_{i=1}^{j-1} f_{(n-i)}^{\sigma}(x)\right)\right\} j=1, . ., n
\end{aligned}
$$

The first agent (1) pays her last feasible amount. The second agent pays her last feasible amount given (1) payment and so on. Each agent is a dictator with respect to the following players. for this reason $f^{\sigma}$ will be called the $\sigma$-serial dictatorship. 
An equivalent definition for $f^{\sigma}$ is

$$
\begin{aligned}
f_{(n)}^{\sigma}(x) & =\min \left\{x_{(n)}, T\right\} \\
f_{(n-j)}^{\sigma}(x) & =\min \left\{x_{(n-j)}, T-\sum_{i=n}^{n-j+1} f_{(n-i)}^{\sigma}(x)\right\} j=1, . ., n-1
\end{aligned}
$$

In words, player $(n)$ pays all amount to be collect if she has enough income. Otherwise what is left is paid by player $(n-1)$ if she has enough income and so on.

Proposition 5. Let $h$ be a non rewarding reversion function and let $f$ be a continuous feasible taxation method. If $f$ is $h$-implementable in then it is a serial dictatorship. If $f$ is a serial dictatorship then it is $h$-implementable for any non rewarding $h$.

Proof Let $x^{\prime} \leq x$. In such a case $T\left(x^{\prime}\right) \subset T(x)$. Let $f(x) \in T\left(x^{\prime}\right)$, which is $f(x) \leq x^{\prime}$ then

$L_{i}(t, x) \cap T(x) \cap T\left(x^{\prime}\right) \subset L_{i}\left(t, x^{\prime}\right)$. If $x^{\prime} \leq x$ and $f(x) \leq x^{\prime}$ then GCC prescribes that $f\left(x^{\prime}\right)=f(x)$. In particular $f(x)=f(y)$ for all $f(x) \leq y \leq x$.

Set $I(t, x)=\{y \in V(x): t \leq y \leq x\}$. Then $f(I(f(x), x)=\{f(x)\}$ for all $x$. Observe that $I(f(x), x) \cap I\left(f\left(x^{\prime}\right), x^{\prime}\right) \neq \varnothing$ implies $f(x)=f\left(x^{\prime}\right)$.

Let $f$ be not a serial dictatorship. Then exists $x, i, j$ such that $0<f_{i}(x)<x_{i}$ and $0<f_{j}(x)<x_{j}$. Then $f(x)$ is in the relative interior of $T(x)$ and $I(f(x), x)$ is full dimensional. Let $y \geq x$. We show that $f(y)=f(x)$. There is no loss of generality in assuming that $f(z) \neq f(x)$ in all $z$ on the segment joining $y$ and $x$. From GCC it follows that for all such $z, f(z) \notin T(x)$. Let $z \rightarrow x$ on this segment then $f(z) \nrightarrow f(x)$ but if $f(z)$ converges then $f(z) \rightarrow t$ where $t$ belongs to the relative boundary of $T(x)$ which contradicts continuity.

Let $x^{*}=(T, \ldots, T)$. It follows that $f(y)=f(x)$ for all $y \geq f(x)$. If $y \nsupseteq f(x)$ then $f(y)=f^{\sigma}(y)$ for some $\sigma$, because otherwise. $I(f(y), y) \cap I\left(f\left(x^{*}\right), x^{*}\right) \neq \varnothing$ and 
$f\left(x^{*}\right)=f(x)$ is not feasible at $y$. But in such a case $f$ would not be continuous.. A contradiction.

The second part of the claim can be proved as in Proposition 4.

Example 2. There are also discontinuous feasible taxation methods that are $h$-implementable in Nash equilibrium as the proof of the previous result suggests. Let $x^{*}=(T, \ldots, T)$, let $t \in \Omega(T)$ and let $\sigma$ be a permutation on $I$.

$$
\begin{aligned}
& f(x)=\left(\frac{T}{n}, \ldots, \frac{T}{n}\right) \text { for all } x \geq\left(\frac{T}{n}, \ldots, \frac{T}{n}\right) \\
& f(x)=f^{\sigma}(x) \text { otherwise }
\end{aligned}
$$

It is not difficult to prove that $f$ is implementable in NE through generalized severe punishment.

\section{Conclusions}

In this paper we have presented a new approach to deal with the implementation problem based on the idea that agents renegotiate unfeasible allocations into feasible ones. We have presented a class of renegotiation functions that are suited to our problem and we have found necessary and sufficient conditions for implementation when renegotiation takes this form. Finally we have used our characterization results to study the implementation in Nash equilibrium of social choice rules in exchange economies, bargaining problems and taxation methods and we have compared our results with those obtained by the earlier literature.

An unnatural feature of the traditional approach of implementation when feasible sets are state dependent is that requires a collection of state dependent mechanisms, contrarily to the case when preferences are state dependent. This distinction contrast 
vividly with our intuition on how markets cope with unfeasible allocations, namely that the sign of excess demand determines entirely the adjustment irrespectively of the cause of infeasibility. ${ }^{13}$ Thus our approach may offer a better understanding of market mechanisms than the traditional one. But on the other hand the traditional approach is better suited to deal with topics like withholding of endowments -in our case the state of the world, and thus endowments, is common knowledge- or tax evasion given the importance of reports in the renegotiation. Actually our approach can be generalized to cope with this cases by introducing uncertainty in the renegotiation process or the mechanism as an argument in the renegotiation function. These two extensions are easy to write but require completely new analytical methods. Thus they are left for future research.

\section{Bibliography}

Amorós, P. "Nash Implementation and Uncertain Renegotiation" Games and Economic Behavior, 49, 2, 424-434.

Benassy, J. P. 1996.

Dagan, N. and R. Serrano "Invariance and randomness in the Nash program for coalitional games" Economics Letters, 58, 43-49

Dagan, N., Serrano, R. and Volij, O. 1999 "Feasible Implementation of Taxation Methods". Review of Economic Design, 4, 57-72.

Hong, L., 1995 "Nash Implementation in Production Economies". Economic Theory, $5,401-417$.

\footnotetext{
${ }^{13}$ In fact, following the lead of Benassy (1986) many papers dealing with markets from the implementation point of view disregard entirely the issue of feasibility.
} 
Hong, L., 1996 "Bayesian Implementation in Exchange Economies with State Dependent Feasible Sets and Private Information". Social Choice and Welfare, 13, 433-444.

Hong L., 1998 "Feasible Bayesian Implementation with State Dependent Feasible Sets." Journal of Economic Theory 80, 201-221

Hurwicz L., Maskin E. and Postlewaite A., 1995 "Feasible Nash Implementation of Social Choice Rules when the Designer does not know Endowments or Production set." in Leydard J. (ed) The Economics of Informational Decentralization: Complexity, Efficiency and Stability, Kluwer Academic Publishing.

Jackson, M. and Palfrey, T., 2001 "Voluntary implementation". Journal of Economic Theory, 98, 1-25.

Maskin, E. and Moore, J. 1999 "Implementation with Renegotiation". Review of Economic Studies, 66, 39-56.

Naeve J., 1999 "Nash implementation of the Nash bargaining solution using intuitive message spaces" Economics Letters, 62, 23-28.

Repullo, R. 1987 "A Simple Proof of Maskin's theorem on Nash Implementation". Social Choice and Welfare, 4, 39-41.

Roemer, J. E. 1996 Theories of Distributive Justice. Harvard University Press.

Serrano R. 1997 "A comment on the Nash program and the theory of implementation" Economics Letters, 55, 203-208

Serrano, R. and Vohra, R. 1997 "Non Cooperative Implementation of the Core". Social Choice and Welfare, 14, 513-525. 
Tian, G. 1993 "Implementing Lindahl Allocations by a Withholding Mechanism". Journal of Mathematical Economics, 22, 163-179

Tian, G., Li, Q. 1995 "On Nash-Implementation in the Presence of Withholding". Games and Economic Behavior, 9, 222-233. 